\title{
OPEN A risk model for detecting clinically significant prostate cancer based on bi-parametric magnetic resonance imaging in a Japanese cohort
}

\begin{abstract}
Kazushige Sakaguchi ${ }^{\bowtie}$, Michikata Hayashida, Naoto Tanaka, Suguru Oka \& Shinji Urakami
Selective identification of men with clinically significant prostate cancer (sPC) is a pivotal issue. Development of a risk model for detecting SPC based on the prostate imaging reporting and data system (PI-RADS) for bi-parametric magnetic resonance imaging (bpMRI) and clinical parameters in a Japanese cohort is expected to prove beneficial. We retrospectively analyzed clinical parameters and bpMRI findings from 773 biopsy-naïve patients between January 2011 and December 2016. A risk model was established using multivariate logistic regression analysis and presented on a nomogram. Discrimination of the risk model was compared using the area under the receiver operating characteristic curve. Statistical differences between the predictive model and clinical parameters were analyzed using DeLong test. SPC was detected in 343 men (44.3\%). Multivariate logistic regression analysis to predict sPC revealed age $(P=0.002)$, log prostate-specific antigen $(P<0.001)$, prostate volume $(P<0.001)$ and PI-RADS scores $(P<0.001)$ as significant contributors to the model. Area under the curve was higher for the risk model $(0.862)$, than for age $(0.646)$, log prostate-specific antigen (0.652), prostate volume $(0.697)$ or imaging score $(0.822)$. DeLong test results also showed that the novel risk model performed significantly better than those parameters $(P<0.05)$. This novel risk model performed significantly better compared with PI-RADS scores and other parameters alone, and is thus expected to prove beneficial in making decisions regarding biopsy on suspicion of SPC.
\end{abstract}

Prostate cancer is the most commonly diagnosed cancer in Japan. The incidence of prostate cancer is rapidly increasing, with over 90,000 men newly diagnosed in 2017 . Over 12,000 men died of prostate cancer in 2018 , representing the 6th-most frequent cause of cancer-related death among men in Japan ${ }^{1}$. Population-based prostatespecific antigen (PSA) screening tests can facilitate early detection of prostate cancer and thus lead to declines in prostate cancer related-mortality ${ }^{2}$. However, these tests simultaneously lack specificity, resulting in increased numbers of unnecessary prostate biopsies, which in turn are associated with risks of rectal bleeding and sepsis. The risk of over-treatment leading to adverse impacts on quality of life without improving survival is a concern. Randomized controlled clinical studies that evaluated the efficacy of prostate cancer screening have highlighted the need to reduce the over-diagnosis of clinically insignificant prostate cancer. A new diagnostic pathway is thus needed to selectively identify men with clinically significant prostate cancer (sPC), while reducing the number of unnecessary biopsies and over-detection and over-treatment of clinically insignificant prostate cancer ${ }^{2,3}$.

The use of multi-parametric magnetic resonance imaging (mpMRI) of the prostate, incorporating anatomical and functional imaging (T2-weighted imaging, diffusion-weighted imaging (DWI) and dynamic contrast enhancement (DCE)) appears beneficial for detecting sPC. However, mpMRI has been criticized for widely variable diagnostic performance across different institutions. In 2012, Prostate Imaging Reporting and Data System (PI-RADS) was introduced to facilitate standardized interpretation of mpMRI findings ${ }^{4}$. In PI-RADS, a score for suspecting the presence of $\mathrm{SPC}$ is assigned on a 5-point scale for the mpMRI sequence. PI-RADS has shown high diagnostic accuracy for detecting sPC by means of targeted biopsies ${ }^{5,6}$.

The use of clinical data with mpMRI findings has become significantly important for urologists to better stratify individuals who may warrant prostate biopsy. Multivariable prediction models are superior to conventional 
decision-making based solely on PSA testing or digital rectal examination (DRE) in predicting the outcomes of prostate biopsies. Previous multivariable prediction models for detecting sPC were based on clinical parameters including various combinations of age, PSA, prostate volume (PV), DRE findings and others. MRI findings were also utilized as a parameter of prediction models, but without a standardized reporting system ${ }^{7,8}$. The utility of an individualized risk calculator and a multivariable nomogram (a nomogram is a graphical calculating device, specifically the approximate probability of sPC derived by mathematical logistic function in this study) including data from mpMRI using PI-RADS score for detecting SPC have been reported ${ }^{9-11}$. Furthermore, the use of bi-parametric MRI (bpMRI) of the prostate incorporating anatomical and functional imaging (T2-weighted imaging and DWI not containing DCE) has been shown to maintain high diagnostic accuracy ${ }^{12,13}$. Predictive models based on bpMRI findings and clinical parameters for risk assessment and selection of sPC have also recently been reported ${ }^{14,15}$.

However, epidemiologically, the characteristics of prostate cancer exhibit regional and ethnic differences ${ }^{16}$. While risk calculators and nomograms should ideally be structured from the same cohorts with good validation, no reports have described a risk calculator and nomogram using PI-RADS scores combined with other clinical parameters from a Japanese-only cohort ${ }^{6}$. The aim of the present study was to develop the first risk model and nomogram using PI-RADS score among Japanese men for detecting sPC and reducing the over-detection and over-treatment of clinically insignificant prostate cancer.

\section{Methods}

Study population. A total of 773 biopsy-naïve patients from a single institution (Toranomon hospital, Tokyo, Japan) between January 2011 and December 2016 and suspected to have localized prostate cancer based on abnormal PSA levels were analyzed retrospectively. Indications for biopsy were high PSA level ( $\geq 4.0 \mathrm{ng} / \mathrm{ml})$, abnormal DRE or lesions suggestive of prostate cancer on bpMRI. Exclusion criteria were previous prostate surgery, previous diagnosis of prostate cancer or administration of 5-alpha-reductase inhibitors or anti-androgens, as agents that affect PSA values. Full data on PI-RADS scores of bpMRI, biopsy outcome, PSA, age and PV were available for all patients. Data from bpMRI performed before the introduction of PI-RADS were reinterpreted and new PI-RADS scores were assigned. Those samples were used for development and internal validation of the risk model. The study was approved by Toranomon Hospital Ethics Committee (approval no. 1573). All methods were conducted in accordance with the relevant local guidelines and regulations. All patients provided informed consent or were informed that the hospital web-page included an opt-out option, as approved by the Toranomon Hospital Ethics Committee.

Imaging. All bpMRI was performed using a 1.5- or 3.0-T system (Magnetom; Siemens, Erlangen, Germany) with a multichannel body surface coil. The bpMRI protocol included axial, coronal and sagittal turbo spin echo T2-weighted sequences and axial DWI with apparent diffusion coefficient (ADC) calculation (Supplementary Table S1). ADC maps were rebuilt in each pixel of each slice using the mono-exponential model. A 1.5-T system was generally used for the first bpMRI and a 3.0-T system was used for the second and subsequent bpMRI. All image analyses were performed according to PI-RADS version 2.0 on a scale from 1 to 5 , with higher numbers indicating a greater likelihood of $\mathrm{sPC}^{17}$. Analyses were performed by or under the supervision of a few expert uroradiologists. Overall, PI-RADS scores for each lesion were determined separately for the peripheral zone and transitional zone, entailing assignment of separate scores for each of the T2-weighted and DWI sequences. PV was calculated on T2-weighted imaging, calculated as $0.52 \times$ length $\times$ width $\times$ height.

Biopsy protocol. All patients underwent systematic transperineal and transrectal biopsy (mapping 8-14 cores) of the whole gland in the lithotomy position under local anesthesia, carried out by one of several expert urologists ${ }^{18}$. The number of needle cores was decided by prostate size and risk of infection associated with rectal biopsy for each patients. If one or more lesions suggestive of prostate cancer were detected on bpMRI (suspicious lesions were consistent with PI-RADS score $\geq 3$ retrospectively), transperineal cognitive targeted biopsies were added for each lesion (2-4 cores of each lesion; median, 2 per lesion). Transrectal ultrasound echography (ARIETTA; Hitachi Aloka Medical, Wallingford, CT, USA) was used to guide biopsies without MRI fusion software.

Histopathology. Histopathological analyses from biopsies were performed by or under the supervision of a few expert uropathologists specializing in prostate assessment according to International Society of Urological Pathology standards. For all cores, the length of the cancer in millimeters and both primary and secondary Gleason grades were assigned separately. The study defined sPC as grade group $\geq 3$ (Gleason score $\geq 4+3$ ) or a maximum cancer core length $\geq 6 \mathrm{~mm}$ in any location ${ }^{5}$.

Statistical analysis. Patient demographics, MRI and biopsy results (age, PSA, PV, PI-RADS score 1-5 and presence or absence of sPC) were analyzed descriptively. First, we divided all patients into two groups by pathological outcome: a sPC group; and an others group. The others group included patients with clinically insignificant prostate cancer or no cancerous tissue. Clinical parameters were compared between groups using the Wilcoxon test and Pearson test. Consequently, we performed multivariate logistic regression analysis to predict the presence of sPC on biopsy. We calculated odds ratios and used multivariate logistic regression-based coefficients to develop multivariable nomograms for predicting the probability of sPC (a nomogram is a graphical calculating device, specifically the approximate probability of SPC derived by mathematical logistic function in this study). To avoid linearity assumptions, PSA was transformed into the logarithmic PSA. 


\begin{tabular}{|l|l|l|r|}
\hline Parameter & sPC group n=343 & Others group n=430 & P value \\
\hline Median age, year (IQR) & $69(64-75)$ & $65(60-70)$ & $<0.001$ \\
\hline Median PSA, ng/ml (IQR) & $9.01(6.32-13.40)$ & $6.72(5.26-9.44)$ & $<0.001$ \\
\hline Median PV, ml (IQR) & $29.60(23.80-37.25)$ & $39.85(29.75-51.77)$ & $<0.001$ \\
\hline Number of cores, median (IQR) & $8(8-14)$ & $8(8-9)$ & 0.021 \\
\hline \multirow{5}{*}{ PI-RADS score } & Score $1 \mathrm{n}=3(0.87 \%)$ & Score $1 \mathrm{n}=8(1.42 \%)$ & \\
\cline { 2 - 3 } & Score $2 \mathrm{n}=19(5.54 \%)$ & Score $2 \mathrm{n}=237(33.12 \%)$ & \multirow{3}{*}{$<0.001$} \\
\cline { 2 - 3 } & Score $3 \mathrm{n}=94(27.41 \%)$ & Score $3 \mathrm{n}=111(26.52 \%)$ & \\
\cline { 2 - 3 } & Score $4 \mathrm{n}=98(28.57 \%)$ & Score $4 \mathrm{n}=44(18.37 \%)$ & \\
\cline { 2 - 3 } & Score $5 \mathrm{n}=129(37.61 \%)$ & Score $5 \mathrm{n}=30(20.57 \%)$ & \\
\hline
\end{tabular}

Table 1. Clinical parameters and MRI PI-RADS score both 2 groups. IQR interquartile range, $P I-R A D S$ prostate imaging reporting and data system, $S A$ prostate-specific antigen, $P V$ prostate volume, $s P C$ clinically significant prostate cancer.

\begin{tabular}{|c|c|c|c|}
\hline Parameter & Odds ratio & 95\% CI & $P$ value \\
\hline Age (per 5 year) & 1.214 & $1.074-1.374$ & 0.002 \\
\hline $\log$ PSA (ng/ml) & 2.101 & $1.441-3.120$ & $<0.001$ \\
\hline $\mathrm{PV}($ per $10 \mathrm{ml})$ & 0.68 & $0.591-0.777$ & $<0.001$ \\
\hline \multicolumn{3}{|l|}{ PI-RADS score } & $<0.001$ \\
\hline PI-RADS score 2: score 1 & 0.292 & $0.073-1.477$ & 0.098 \\
\hline PI-RADS score 3: score 1 & 2.005 & $0.532-9.725$ & 0.332 \\
\hline PI-RADS score 4: score 1 & 4.694 & $1.220-23.11$ & 0.033 \\
\hline PI-RADS score 5: score 1 & 6.178 & $1.552-31.16$ & 0.014 \\
\hline
\end{tabular}

Table 2. Multivariate logistic regression model analysis for the prediction of sPC. CI confidence interval, $P I-R A D S$ prostate imaging reporting and data system, $P S A$ prostate-specific antigen, $P V$ prostate volume, $s P C$ clinically significant prostate cancer.

The discrimination of risk models for sPC with or without MRI scoring was compared using the area under the curve (AUC) of receiver operating characteristic (ROC) curves. The significance of differences between predictive models was analyzed using DeLong test.

The extent of over- or underestimation of the predicted rate relative to the observed rate of sPC was explored graphically using calibration plots, which were internally validated using 1000 bootstrap resamples. The intercept indicates whether predictions are systematically too low or too high, and thus should ideally be zero. The calibration slope reflects the average effects of predictors in the model and is estimated in a logistic regression model with the logit of model predictions as the only predictor. For a perfect model, the slope equals $1^{19}$.

Last, we assessed the performance of the risk model for its clinical usefulness using decision curve analysis (DCA) based on bootstrapped validation repeated 1000 times. These analyses estimate a 'net benefit' for prediction models by totaling the benefits (true-positive biopsies) and subtracting the harms (false-positives biopsies) ${ }^{20}$. Harms are weighted by the relative harm of a missed sPC compared to unnecessary biopsy. The weighted rate is derived from the threshold probability of sPC at which a patient would opt for biopsy. This threshold can thus vary from patient to patient in clinical settings. The reduction in number of biopsies using different probabilities was further assessed and related to the number and percentage of sPC detected. Interpretation of the decision curve was based on the model with the highest net benefit at a particular threshold probability representing the most useful model for risk and benefit. To quantify the potential reduction of unnecessary biopsies and potential over-diagnosis, we calculated true-positive rate (TPR), false-positive rate (FPR), positive predictive value (PPV) and negative predictive value (NPV) at exemplary probability thresholds.

All tests performed were two-sided and values of $P<0.05$ were considered to indicative of statistical significance. Statistical analyses were performed using R version 4.0.2 (R Foundation for Statistical Computing, Vienna, Austria). ROC analysis and DCA were performed utilizing the pROC package and rmda package, respectively. Reporting followed the Standards of Reporting of Diagnostic Accuracy (Supplementary Tables S2, S3).

\section{Results}

In total, sPC was detected in 343 men (44.3\%). Demographic characteristic, and MRI and biopsy data from both groups are given in Table 1. Men in the sPC group were older (median age, 69 years vs 65 years, $P<0.001$ ), had higher PSA (median PSA, $9.01 \mathrm{ng} / \mathrm{ml}$ vs $6.72 \mathrm{ng} / \mathrm{ml}, P<0.001$ ), and had lower PV (median PV, $29.6 \mathrm{ml}$ vs $39.85 \mathrm{ml}, P<0.001$ ). The proportion of borderline and malignant lesions on bpMRI (PI-RADS score 3, 4 or 5) was significantly higher in the sPC group $(93.59 \%$ vs $65.46 \%, P<0.001)$.

Multivariate logistic regression analysis to predict sPC identified age $(P=0.002), \log P S A(P<0.001), \mathrm{PV}$ $(P<0.001)$ and PI-RADS score $(P<0.001)$ as significant contributors to the model (Table 2$)$. Multicollinearity 
Points

$\begin{array}{lllllllllll}0 & 10 & 20 & 30 & 40 & 50 & 60 & 70 & 80 & 90 & 100\end{array}$

Age

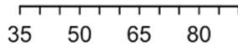

MRI.PV

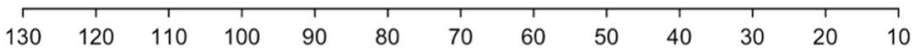

PSA

category

Total Points

Significant cancer
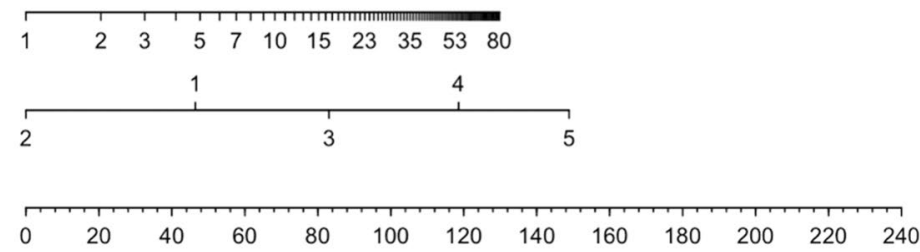

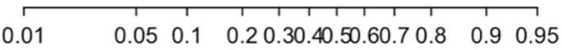

Figure 1. Risk model to predict sPC including age, PSA, PV and bpMRI PI-RADS score .

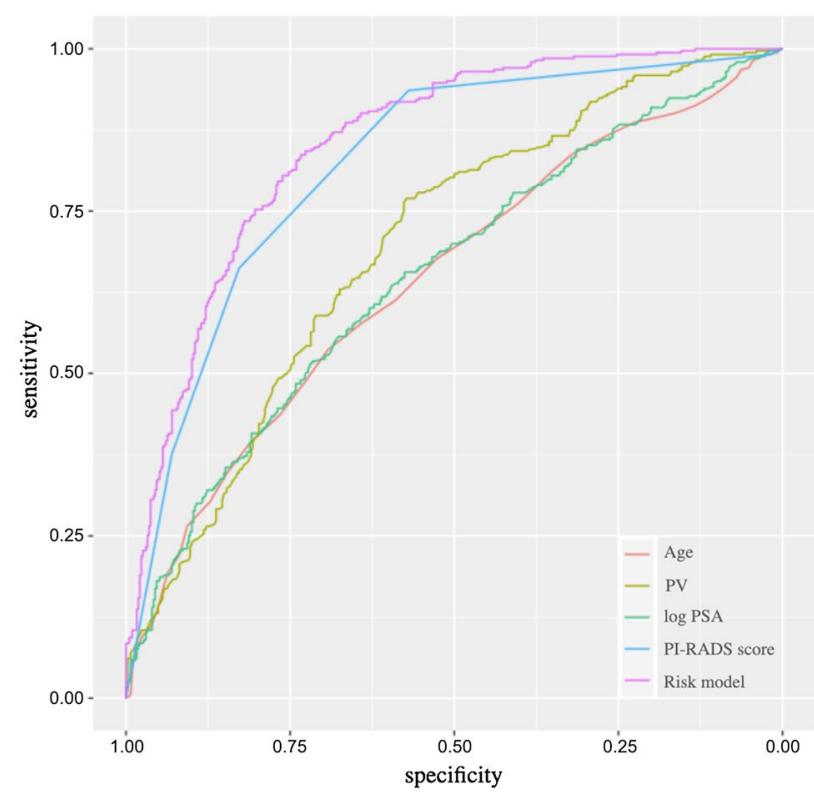

Figure 2. ROC curve analysis for the performance of age (red line), PV (yellow line), PSA (green line), bpMRI PI-RADS (blue line) and risk model (purple line) to predict sPC.

was tested between all variables by the individual variance inflation factor and no multicollinearity was found. The nomogram of the risk model and the regression equation are shown in Fig. 1.

The novel risk model was internally validated by bootstrapping. Discrimination of the risk model was compared using parameters included in ROC analyses (Fig. 2, Table 3). AUC was higher for the risk model (0.862), than for age (0.646), PV (0.697), logPSA (0.652) or PI-RADS score (0.822). DeLong test results also showed that the novel risk model performed significantly better compared with those parameters including PI-RADS score alone (Table 3). Table4 shows TPR, FPR, PPV and NPV at exemplary probability thresholds of this risk model and the optimal PI-RADS score cutoff. At a probability threshold of $10 \%$, the net reduction in biopsies taken based on the risk model was $43.0 \%$, while the rate of missing sPC was $2.3 \%$. Bootstrapped calibration plots of the risk model demonstrated no untoward deviations of predicted risk from observed risk of sPC over the entire range (Fig. 3).

In bootstrapped DCA, the risk model showed a higher net benefit in terms of accurately detecting patients with sPC, compared with PI-RADS score and other parameters alone (Fig. 4). The risk model showed a benefit for sPC threshold probabilities larger than $10 \%$. 


\begin{tabular}{|c|c|c|}
\hline & AUC & $95 \% \mathrm{CI}$ \\
\hline \multicolumn{3}{|l|}{ Parameter } \\
\hline Age & 0.646 & $0.607-0.685$ \\
\hline $\mathrm{PV}$ & 0.697 & $0.661-0.734$ \\
\hline $\log$ PSA & 0.652 & $0.613-0.691$ \\
\hline PI-RADS score & 0.822 & $0.793-0.851$ \\
\hline \multirow[t]{2}{*}{ Risk model } & 0.862 & $0.837-0.888$ \\
\hline & $P$ value & \\
\hline \multicolumn{3}{|l|}{ Comparison of models and factors } \\
\hline Risk model versus Age & $<0.001$ & \\
\hline Risk model versus PV & $<0.001$ & \\
\hline Risk model versus log PSA & $<0.001$ & \\
\hline Risk model versus PI-RADS score & 0.039 & \\
\hline
\end{tabular}

Table 3. AUC of ROC curve analysis for the performance of age, PV, PSA, PI-RADS and risk model to $\mathrm{SPC}$, and DeLong test for model and factors comparison. AUC area under the curve, CI confidence interval, $P I-R A D S$ prostate imaging reporting and data system, $P S A$ prostate-specific antigen, $P V$ prostate volume, $R O C$ reciever operating characteristic, $s P C$ clinically significant prostate cancer.

\begin{tabular}{|l|l|l|l|l|}
\hline Risk model & TPR & FPR & PPV & NPV \\
\hline Parameter & \multicolumn{5}{l|}{} \\
\hline 5\% probability of sPC cut-off & 0.994 & 0.811 & 0.496 & 0.976 \\
\hline $10 \%$ probability of sPC cut-off & 0.977 & 0.57 & 0.579 & 0.958 \\
\hline $20 \%$ probability of sPC cut-off & 0.936 & 0.43 & 0.636 & 0.917 \\
\hline $50 \%$ probability of sPC cut-off & 0.787 & 0.217 & 0.744 & 0.821 \\
\hline Best cut-off of risk model: 41\% probability of sPC & 0.863 & 0.271 & 0.718 & 0.869 \\
\hline Best cut-off of PI-RADS: score between 2 and 3 & 0.936 & 0.43 & 0.634 & 0.918 \\
\hline
\end{tabular}

Table 4. Prediction errors for diagnosis of sPC as 5\%,10\%,20\%,50\% and best cut-offs for risk model and PI-RADS score . FPR false positive rate, NPV negative predictive value, $P I-R A D S$ prostate imaging reporting and data system, $P P V$ positive predictive value, $s P C$ clinically significant prostate cancer, $T P R$ true positive rate.

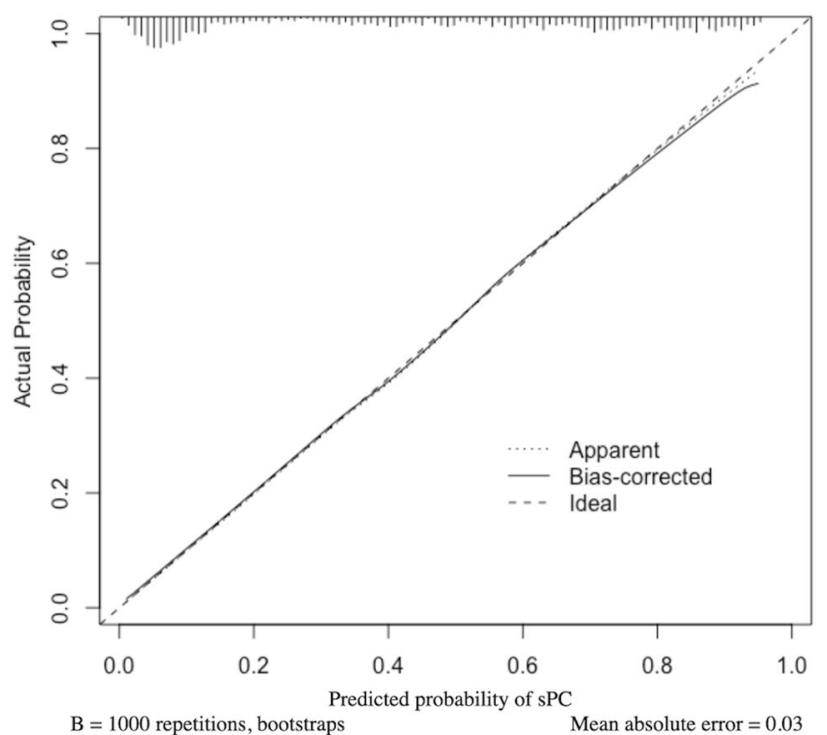

Figure 3. Calibration plots for the risk models to predict sPC. 


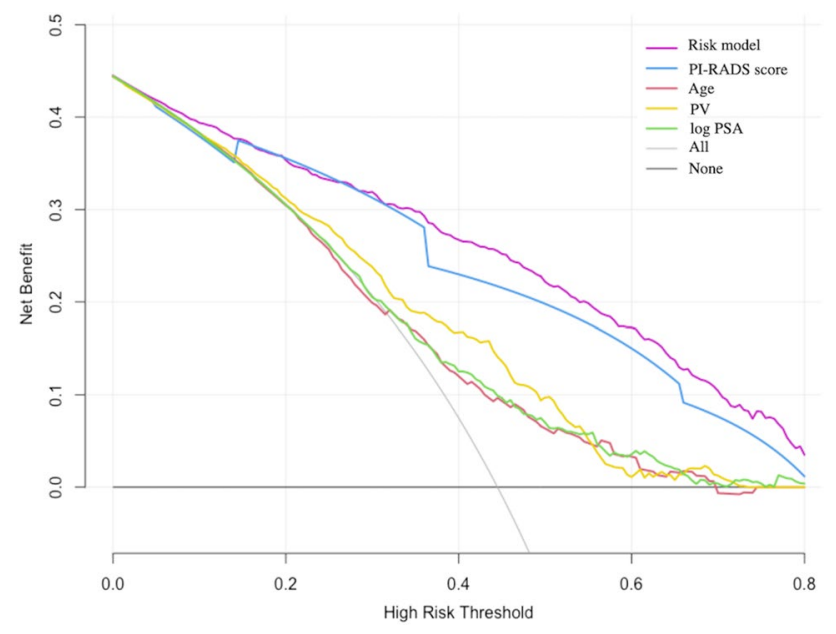

Figure 4. Net DCA demonstrating the benefit for predicting sPC on biopsy.

\section{Discussion}

While mpMRI can detect $85-95 \%$ of sPC compared with prostatectomy specimens, the sensitivity, NPV and specificity of mpMRI have been reported as $58-96 \%, 63-98 \%$ and $23-87 \%$, respectively ${ }^{5,21}$. Because of the high diagnostic accuracy for sPC detection, upfront mpMRI has been recommended as a triage test to indicate the need for biopsy among biopsy-naïve men in whom $\mathrm{PPC}$ was suspected due to high $\mathrm{PSA}^{22-24}$. As a result of the high NPV, men with no suspected evidence of sPC on MRI may defer systematic biopsy ${ }^{25}$. Moreover, to improve predictive values, new multivariate risk prediction tools have recently been constructed using the mpMRI suspicion score ${ }^{9,10,26}$.

The bpMRI procedure involves performing prostate MRI without DCE, and produces beneficial results. The effectiveness of bpMRI detecting sPC in biopsy-naïve patients has been reported. In addition, bpMRI has the advantage of avoiding the adverse events associated with some gadolinium-based contrast agents, as well as shortened examination time and reduced costs ${ }^{27}$. On the other hand, DCE MRI has been reported to improve the sensitivity of MRI for detecting sPC. At the same time, predictive models based on bpMRI findings and clinical parameters for risk assessment and selection of sPC have also been reported recently ${ }^{14,15,28,29}$.

In a Japanese cohort, the efficacy of mpMRI and bpMRI for detecting $s$ PC as a triage test has been reported $^{30-32}$. However, no multivariate risk prediction models for detecting sPC based on PI-RADS scores of mpMRI or bpMRI as ordinal variables among Japanese populations have been described previously.

The characteristics of our novel risk model were as follows. First, in all cases, bpMRI was performed on the pre-biopsy setting, because biopsy artifacts could affect bpMRI findings and this model was constructed to reduce unnecessary biopsies. Second, variables of DRE and PSA density used in other nomograms were not included in this study. Because anterior prostate cancer is less commonly palpable, use of DRE as a variable in the prediction model means that the dataset of the model should ideally be divided into two groups according to whether DRE findings are positive or not, and each model should be constructed independently ${ }^{33}$. The small size of our dataset did not allow division into two groups. The parameters PSA and PV, and not PSA density, were selected because their interpretations are more explainable and understandable 9 .

PI-RADS score contributed significantly to the model, like other parameters from multivariate logistic regression analysis. Interestingly, the odds ratio of PI-RADS score 2 compared to score 1 was $0.292(P=0.098)$, while that of PI-RADS score 3 compared to score 1 was $2.005(P=0.332)$ (Table 2). PI-RADS score 1 and score 2 indicated a normal prostate gland and benign prostate disease (inflammatory and/or hyperplasia), respectively. In a proportion of cases with PI-RADS score 2, PSA was elevated because of inflammation and hyperplasia. Therefore, among high-PSA cases, PI-RADS score 1 might carry a higher risk of sPC than PI-RADS score 2 in real clinical practice. Moreover, because of the low number of cases with PI-RADS score 1 (only 11 cases, $1.42 \%$ ), the odds ratio for PI-RADS score 2 to score 1 might not reach statistical significance. This also explained why lower PV cases tended to carry a higher risk of sPC. This was presumably because multicollinearity among parameters could not be completely excluded even if multivariate analysis was performed.

A low PI-RADS score harbors a 5-10\% risk of sPC, allowing biopsy to be potentially avoided ${ }^{21,34}$. Multivariate risk prediction tools including mpMRI findings from regions other than Japan have shown a high AUC of $0.82-0.91^{35}$. ROC analysis revealed that this novel model offered a high AUC (c index $=0.862$ ) approximately equivalent to previous reports, although this novel model has not been externally validated and should not be compared to other risk models constructed from different regional and ethnic cohorts ${ }^{9}$. The risk model enables avoidance of unnecessary biopsies in more patients without increasing the risk of missing a diagnosis of sPC at an arbitrary probability threshold. More specifically, at probability thresholds of $10 \%$ and $20 \%$ in this model and with a cut-off PI-RADS score between 2 and 3, net reductions in biopsies were $43.0 \%, 57.0 \%$ and $57.0 \%$, while rates of missing sPC were $2.3 \%, 6.4 \%$ and $6.4 \%$, respectively. Using DCA, the present study showed that the risk model using PI-RADS scores improved clinical decisions for the biopsy of patients with suspected sPC, as compared with clinical parameter models or PI-RADS score alone. The risk model provided benefits in the 
decision to biopsy patients for sPC at probability thresholds exceeding $10 \%$. From a practical perspective, at various probability cutoffs, the combined models demonstrated the best performance among all prediction parameters. Although cost-effectiveness remains an issue due to differences in social insurance situations and the high penetration rate of MRI in other countries, a protocol for biopsy indications for MRI in cases with high PSA value should be considered.

The present findings should be interpreted in the context of some limitations. First, this study represented a retrospective analysis, thus elevating the risk of selection biases. Second, inter-reader agreement on bpMRI was not evaluated in the present study. Third, low numbers of systemic biopsy cores were collected in our cohort. The number of sPC lesions detected by systemic biopsy was thought to be lower and could have improved model accuracy and internal validation. Fourth, this study targeted the first biopsy cases and the findings thus are not applicable to repeat biopsy cases. Last, no external validation was performed. If the excellent results obtained with bpMRI and other clinical parameters from a single institution as in this study are not reproduced in other hospitals, the broad use of the novel risk model will lead to patient mismanagement in a substantial proportion of cases.

To the best of our knowledge, this represents the first report of a risk calculator and nomogram using PIRADS version 2 score of bpMRI among Japanese males for detecting sPC in pre-biopsy settings. On the other hand, recent risk models have been reported to detect sPC using quantitative mpMRI, which may also help standardize mpMRI and bpMRI interpretation and image recognition using new statistical tools (machine learning, deep learning and neural network analysis) ${ }^{36,37}$. Risk models using genetic elements and molecular markers rather than image variables are also being reported ${ }^{38}$. Finally, prospective and multi-centric risk models for sPC risk prediction including such new biochemical parameters, financial aspects and novel MRI fusion biopsy data are expected to be established in the future.

Received: 8 May 2021; Accepted: 6 September 2021

Published online: 22 September 2021

\section{References}

1. Cancer Registry and Statistics. Cancer Information Service, National Cancer Center, Japan, 2018 (2018). https://ganjoho.jp/reg stat/statistics/stat/summary.html. Accessed 22 November 2020.

2. Schröder, F. H. et al. Screening and prostate cancer mortality: Results of the European Randomised Study of Screening for Prostate Cancer (ERSPC) at 13 years of follow-up. Lancet 384, 2027-2035 (2014).

3. Heijnsdijk, E. A. et al. Quality-of-life effects of prostate-specific antigen screening. N. Engl. J. Med. 367, 595-605 (2012).

4. Barentsz, J. O. et al. ESUR prostate MR guidelines 2012. Eur. Radiol. 22, 746-757 (2012).

5. Ahmed, H. U. et al. Diagnostic accuracy of multi-parametric MRI and TRUS biopsy in prostate cancer (PROMIS): A paired validating confirmatory study. Lancet $\mathbf{3 8 9}, 815-822$ (2017).

6. Rouviere, O. \& Moldovan, P. C. The current role of prostate multi parametric magnetic resonance imaging. Asian J. Urol. 6, 137-145 (2019).

7. Nam, R. K. et al. Assessing individual risk for prostate cancer. J. Clin. Oncol. 25, 3582-3588 (2007).

8. Karakiewicz, P. I. et al. Development and validation of a nomogram predicting the outcome of prostate biopsy based on patient age, digital rectal examination and serum prostate specific antigen. J. Urol. 173, 1930-1934 (2005).

9. Radtke, J. P. et al. Combined clinical parameters and multiparametric magnetic resonance imaging for advanced risk modeling of prostate cancer-patient-tailored risk stratification can reduce unnecessary biopsies. Eur. Urol. 72, 888-896 (2017).

10. Mehralivand, S. et al. A magnetic resonance imaging-based prediction model for prostate biopsy risk stratification. JAMA Oncol. 4, 678-685 (2018).

11. Ippolito, D. et al. Diagnostic accuracy of multi parametric magnetic resonance imaging combined with clinical parameters in the detection of clinically significant prostate cancer: A novel diagnostic model. Int. J. Urol. 27, 866-873 (2020).

12. Boesen, L. et al. Assessment of the diagnostic accuracy on biparametric magnetic resonance imaging for prostate cancer in biopsynaive men the biparametric MRI for detection of prostate cancer (BIDOC) Study. JAMA Netw. Open 1, e180219 (2018).

13. Sherrer, R. L. et al. Comparison of biparametric MRI to full multiparametric MRI for detection of clinically significant prostate cancer. Prostate Cancer Prostatic Dis. 22, 331-336 (2019).

14. Bosen, L. et al. A predictive model based on biparametric magnetic resonance imaging and clinical parameters for improved risk assessment and selection of biopsy-naive men for prostate biopsies. Prostate Cancer Prostatic Dis. 22, 609-616 (2019).

15. Perez, I. M. et al. Qualitative and quantitative reporting of a unique biparametric MRI: Towards biparametric MRI-based nomogram for prediction of prostate biopsy outcome in men with a clinical suspicion of prostate cancer (IMPROD and MULTI-IMPROD trials). J. Magn. Reson. Imaging. 51, 1556-1567 (2020).

16. Kimura, T. \& Egawa, S. Epidemiology of prostate cancer in Asian countries. Int. J. Urol. 25, 524-531 (2018).

17. American College of Radiology. MR Prostate Imaging Reporting and Data System Version 2.0 (2015). http://www.acr.org/Quali ty-Safety/Resources/PIRADS/. Accessed 17 October 2017

18. Kawakami, S. et al. Three-dimensional combination of transrectal and transperineal biopsies for efficient detection of stage T1c prostate cancer. Int. J. Clin. Oncol. 11, 127-132 (2006).

19. Moons, K. G. M. et al. Transparent reporting of a multivariable prediction model for individual prognosis or diagnosis (TRIPOD): Explanation and elaboration. Ann. Intern. Med. 162, W1-W73 (2015).

20. Vickers, A. J. \& Elkin, E. V. Decision curve analysis: A novel method for evaluating prediction models. Med. Decis. Mak. 26, 565-574 (2006).

21. Radtke, J. P. et al. Multiparametric magnetic resonance imaging (MRI) and MRI-transrectal ultrasound fusion biopsy for index tumor detection: Correlation with radical prostatectomy specimen. Eur. Urol. 70, 846-853 (2016).

22. European Association of Urology (EAU). Guidelines on Prostate Cancer (2020). http://uroweb.org/guideline/prostate-cancer/. Accessed 22 November 2020.

23. Rouviere, O. et al. Use of prostate systematic and targeted biopsy on the basis of multiparametric MRI in biopsy-naive patients (MRI-FIRST): A prospective, multicentre, paired diagnostic study. Lancet Oncol. 20, 100-109 (2019).

24. van der Leest, M. et al. Head-to-head comparison of transrectal ultrasound-guided prostate biopsy versus multiparametric prostate resonance imaging with subsequent magnetic resonance-guided biopsy in biopsy-naive men with elevated prostate specific antigen: A large prospective multicenter clinical study. Eur. Urol. 75, 570-578 (2019). 
25. Padhani, A. R. et al. Prostate imaging reporting and data system steering committee: PI-RADS v2 status update and future directions. Eur. Urol. 75, 385-396 (2019).

26. van Leeuwen, P. J. et al. A multiparametric magnetic resonance imaging-based risk model to determine the risk of significant prostate cancer prior to biopsy. BJU Int. 120, 774-781 (2017).

27. Turkbey, B. et al. Prostate imaging reporting and data system version 2.1: 2019 update of prostate imaging reporting and data system version 2. Eur. Urol. 76, 340-351 (2019).

28. Greer, M. D. et al. Validation of the dominant sequence paradigm and role of dynamic contrast-enhanced imaging in PI-RADS version 2. Radiology 285, 859-869 (2017).

29. Rosenkrantz, A. B. et al. Proposed adjustments to PI-RADS version 2 decision rules: Impact on prostate cancer detection. Radiology 283, 119-129 (2017).

30. Numao, N. et al. Usefulness of pre-biopsy multiparametric magnetic resonance imaging and clinical variables to reduce initial prostate biopsy in men with suspected clinically localized prostate cancer. J. Urol. 190, 502-508 (2013).

31. Washino, S. et al. Combination of prostate imaging reporting and data system (PI-RADS) score and prostate-specific antigen (PSA) density predicts biopsy outcome in prostate biopsy naive patients. BJU Int. 119, 225-233 (2017).

32. Tamada, T. et al. Comparison of biparametric and multiparametric MRI for clinically significant prostate cancer detection with PI-RADS version 2.1. J. Magn. Reson. Imaging 53, 283-291 (2021).

33. Kim, M. et al. Characteristics of anteriorly located prostate cancer and the usefulness of multi parametric magnetic resonance imaging for diagnosis. J. Urol. 196, 367-373 (2016).

34. Meng, X. et al. Relationship between prebiopsy multiparametric magnetic resonance imaging (MRI), biopsy indication, and MRIultrasound fusion-targeted prostate biopsy outcomes. Eur. Urol. 69, 512-517 (2016).

35. Schoots, I. G. \& Roobol, M. J. Multivariate risk prediction tools including MRI for individualized biopsy decision in prostate cancer diagnosis: Current status and future directions. World J. Urol. 38, 517-529 (2020).

36. Zhang, Y. et al. Development of a novel, multi-parametric, MRI-based radiomic nomogram for differentiating between clinically significant and insignificant prostate cancer. Front. Oncol. 10, 888 (2020).

37. Arif, M. et al. Clinically significant prostate cancer detection and segmentation in low-risk patients using a convolutional neural network on multi-parametric MRI. Eur. Radiol. 30, 6582-6592 (2020).

38. Pepe, P., Dibenedetto, G., Pepe, L. \& Pennisi, M. Multiparametric MRI versus SelectMDx accuracy in the diagnosis of clinically significant PCa in men enrolled in active surveillance. In Vivo 34, 393-396 (2020).

\section{Author contributions}

Conception and design: K.S. Acquisition of data: K.S., M.H. and N.T. Analysis and Interpretation of data, Drafting of the manuscript and Statistical analysis: K.S. Supervision: S.U. All authors reviewed the manuscript.

\section{Competing interests}

The authors declare no competing interests.

\section{Additional information}

Supplementary Information The online version contains supplementary material available at https://doi.org/ 10.1038/s41598-021-98195-2.

Correspondence and requests for materials should be addressed to K.S.

Reprints and permissions information is available at www.nature.com/reprints.

Publisher's note Springer Nature remains neutral with regard to jurisdictional claims in published maps and institutional affiliations.

(c) (i) Open Access This article is licensed under a Creative Commons Attribution 4.0 International License, which permits use, sharing, adaptation, distribution and reproduction in any medium or format, as long as you give appropriate credit to the original author(s) and the source, provide a link to the Creative Commons licence, and indicate if changes were made. The images or other third party material in this article are included in the article's Creative Commons licence, unless indicated otherwise in a credit line to the material. If material is not included in the article's Creative Commons licence and your intended use is not permitted by statutory regulation or exceeds the permitted use, you will need to obtain permission directly from the copyright holder. To view a copy of this licence, visit http://creativecommons.org/licenses/by/4.0/.

(C) The Author(s) 2021 